\title{
Microbiote \\ intestinal \\ et développement \\ des maladies \\ inflammatoires \\ chroniques de \\ l'intestin
}

> Ces dernières années, l'importance d'un microbiote

intestinal bien équilibré (eubiose) est devenue de plus en plus évidente pour la bonne santé de l'homme. L'existence d'une dysbiose est ainsi une caractéristique des patients atteints de maladies inflammatoires chroniques de l'intestin (MICI). Cette altération de l'équilibre de la microflore normale peut avoir plusieurs origines: (1) une alimentation trop riche en graisses d'origine animale et trop pauvre en fibres; (2) une rupture brutale de l'équilibre à la suite d'une gastroentérite aiguë, ou induite par une antibiothérapie; (3) une accumulation, dans la petite enfance, d'événements qui interfèrent avec l'établissement de l'eubiose intestinale (I'hypothèse d'hygiène). Chacune de ces causes peut représenter un facteur de risque de développer une $\mathrm{MICl}$. Une dysbiose peut également faciliter la colonisation de l'intestin par différents pathobiontes capables d'aggraver l'inflammation intestinale. L'AIEC (Escherichia coli adhérent et invasif) représente l'une des bactéries supposées avoir un rôle important dans le développement des $\mathrm{MICl}$. <

La maladie de Crohn ( $M C$ ) et la rectocolite hémorragique $(\mathrm{RCH})$ sont deux maladies inflammatoires chroniques de l'intestin ( $\mathrm{MICl}$ ). Elles se caractérisent par une inflammation de la paroi d'une partie du tube digestif liée à une hyperactivation du système immunitaire digestif. Bien que l'étiologie des $\mathrm{MICl}$ reste encore méconnue, plusieurs facteurs de risque sont suspectés, notamment des facteurs génétiques et environnementaux [1]. L'analyse du génome de patients atteints de $\mathrm{MICl}$ a en effet permis d'identifier plus de 150 gènes de prédisposition à ces maladies. Ils correspondent à un large éventail de gènes impliqués dans différents mécanismes physiopathologiques comme la reconnaissance de microbes, l'activation des lymphocytes, la signalisation et la production de cytokines, et la défense de l'épithélium intestinal (Figure 1) [2-4].

Vignette (Photo @ Inserm - Thierry Pédron).

\author{
Oumaira Rahmouni, Laurent Dubuquoy, \\ Pierre Desreumaux, Christel Neut
}

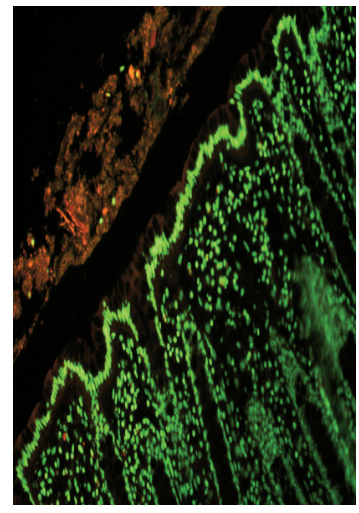

LIRIC UMR 995 Inserm ; université de Lille ; CHRU de Lille, faculté de médecine, Place de Verdun, F-59045, Lille Cedex, France. christelneut@nordnet.fr

À côté de ces gènes de susceptibilité, des données récentes ont suggéré un rôle de la microflore intestinale dans le développement, le maintien, et l'aggravation des $\mathrm{MICl}$ [5]. Bien qu'il soit désormais bien admis que les $\mathrm{MICl}$ résultent d'un défaut d'interaction entre la flore intestinale et le système immunitaire, la nature précise du déséquilibre de la flore intestinale (dysbiose) à l'origine de ce défaut demeure méconnue [6]. Un grand nombre d'hypothèses ont été évoquées pour expliquer et comprendre le rôle de la microflore intestinale dans les $\mathrm{MICI}$, notamment la présence de pathogènes spécifiques et opportunistes, une dysbiose et/ou des altérations fonctionnelles de la flore commensale, ou encore l'implication de défauts génétiques de l'hôte touchant ses capacités de réponse vis-à-vis de la flore commensale [6].

Le microbiote intestinal est un écosystème extrêmement complexe. II compte environ $10^{14}$ microorganismes, recouvrant plus de 1500 espèces bactériennes. Cet écosystème se forme très rapidement après la naissance. Le nouveau-né, stérile, se colonise avec une flore qui est le résultat du contact avec sa mère et avec son environnement. C'est avec la diversification alimentaire que la composition de la flore s'accroît à la fois en diversité (multipliant les espèces) et en richesse pour se stabiliser vers l'âge de 3 ans (Figure 2A). On qualifiera d'eubiose l'état d'un microbiote intestinal «normal » et «équilibré ». Remplissant différentes fonctions métaboliques, d'immunité et de barrière, il permettra le maintien d'un état de bonne santé $[24,25](\rightarrow)$.

$(\rightarrow)$ Voir les Synthèse de R. Burcelin et al., et de V. GaboriauRouthiau et N. CerfBensussan, pages 952 et 961 de ce numéro 


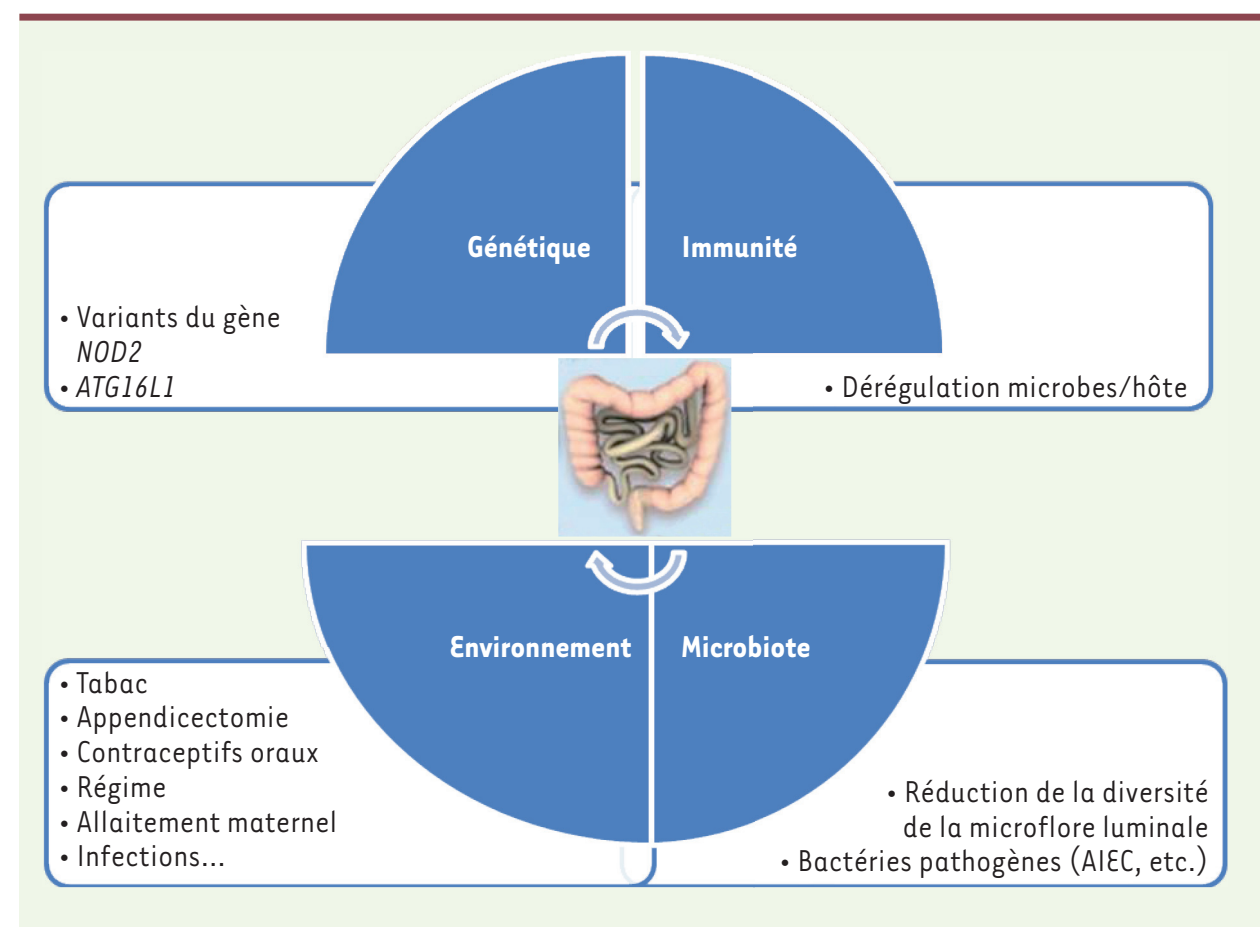

Figure 1. Maladies inflammatoires chroniques de l'intestin (MICl). Les $\mathrm{MICl}$ sont des pathologies complexes pour lesquelles les facteurs environnementaux, la génétique et la microflore intestinale entraîneraient une suractivation du système immunitaire et conduiraient à une inflammation chronique de l'intestin. AIEC : Escherichia coli adhérent et invasif; NOD2 : nucleotide-binding oligomerization domain 2 ; ATG16L1 : autophagy related 16 like 1 (d'après [2-4]).

observé lors de la consommation excessive de sucres et édulcorants, de bonbons, de graisses et d'huile, et de matières grasses totales [3]. Le régime alimentaire «occi-

La composition du microbiote, une fois qu'il est constitué, reste, pour un même sujet, relativement stable au cours de sa vie.

Beaucoup d'arguments sont en faveur de l'apparition chez les patients atteints de $\mathrm{MICl}$, d'une dysbiose bien avant les premiers signes de la maladie [7]. Les principaux facteurs de risque de développer la MC, que nous allons aborder, sont ainsi liés à des facteurs qui influencent la composition de la microflore intestinale [3].

\section{La dysbiose intestinale, un terrain propice au déclenchement d'une $\mathrm{MICl}$}

La dysbiose intestinale peut être induite par au moins trois mécanismes différents.

\section{L’hypothèse diététique}

Les pays développés ou «occidentalisés » sont à forte incidence et prévalence de $\mathrm{MICl}$. Le régime alimentaire «occidental » (riche en graisses et en protéines et pauvre en fibres) prédominant dans les pays de sur-incidence de $\mathrm{MICl}$, peut perturber la microflore intestinale. Les migrants en provenance de pays à faible incidence de $\mathrm{MICl}$ pourront présenter un risque accru de développer des $\mathrm{MICl}$ lorsqu'ils auront adopté les attitudes alimentaires « occidentales».

Certains aliments ont été associés aux $\mathrm{MICl}$. Ils incluent la viande, les graisses, les huiles, la confiserie, et la restauration rapide. Les fibres, les fruits et les légumes ont quant à eux, un rôle de protection contre le développement de ces maladies (Figure 2B). Les régimes riches en saccharose, glucides raffinés, et acides gras oméga- 6 polyinsaturés associés à un régime à faible teneur en fruits et légumes conduisent à un risque accru de développer une $\mathrm{MICl}$, en particulier la $\mathrm{MC}$ [8]. De même, un risque deux fois plus important de développer une MC a été dentalisé », riche en graisses et glucides, favorise l'expansion de pathobiontes ${ }^{1}$ dans le tractus gastro-intestinal, ce qui conduit à une diminution de la représentation des bactéries commensales. Les pathobiontes émergents, de par leur métabolisme, exposent ainsi l'hôte à des constituants néfastes qu'ils produisent (comme le sulfure d’hydrogène) et réduisent des produits bénéfiques produits par les autres bactéries comme les acides gras à chaînes courtes (AGCC). Ils induisent également une réponse immunitaire, provoquant la production de cytokines pro-inflammatoires par les cellules présentatrices d'antigènes (IL[interleukine]$12 \mathrm{p} 40^{2}$ ) et les lymphocytes T (IFN[interféron]-gamma) augmentant ainsi l'inflammation intestinale [8]. Une association entre $M C$ et consommation importante en graisses totales, en acides gras monoinsaturés et saturés a également été observée. Ce régime alimentaire modulerait la réponse inflammatoire en interférant avec les TLR (tolllike receptor) exprimés par les macrophages [3].

L'ingestion de nourriture raffinée, contenant des microparticules, est typique du régime alimentaire «occidental ». Des microparticules comme le dioxyde de titane et le silicate d'aluminium peuvent agir comme des transporteurs d'antigènes de la lumière intestinale vers la muqueuse. Une relation entre la présence de ces microparticules et la MC a été évoquée bien que cela reste controversé [10].

\footnotetext{
${ }^{1}$ Membres agressifs normalement sous-dominants de la flore commensale.

${ }^{2}$ Cette protéine est également appelée «sous-unité bêta de l'interleukine-12 », régulateur important de la réponse inflammatoire.
} 
A
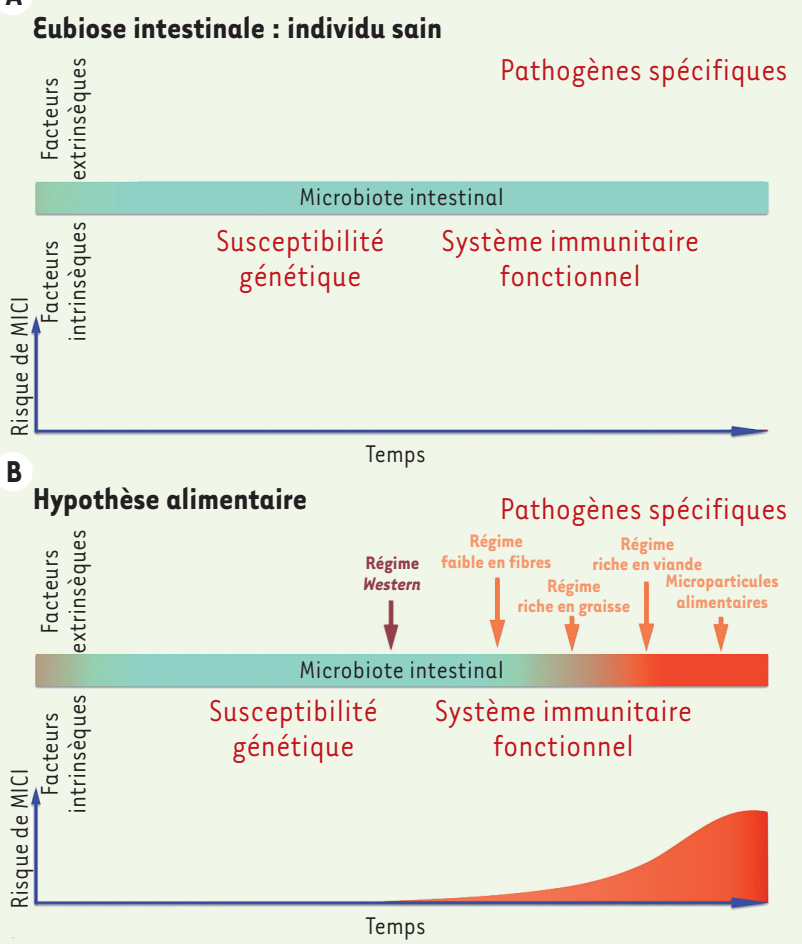

C Rupture aiguë de l'équilibre intestinal

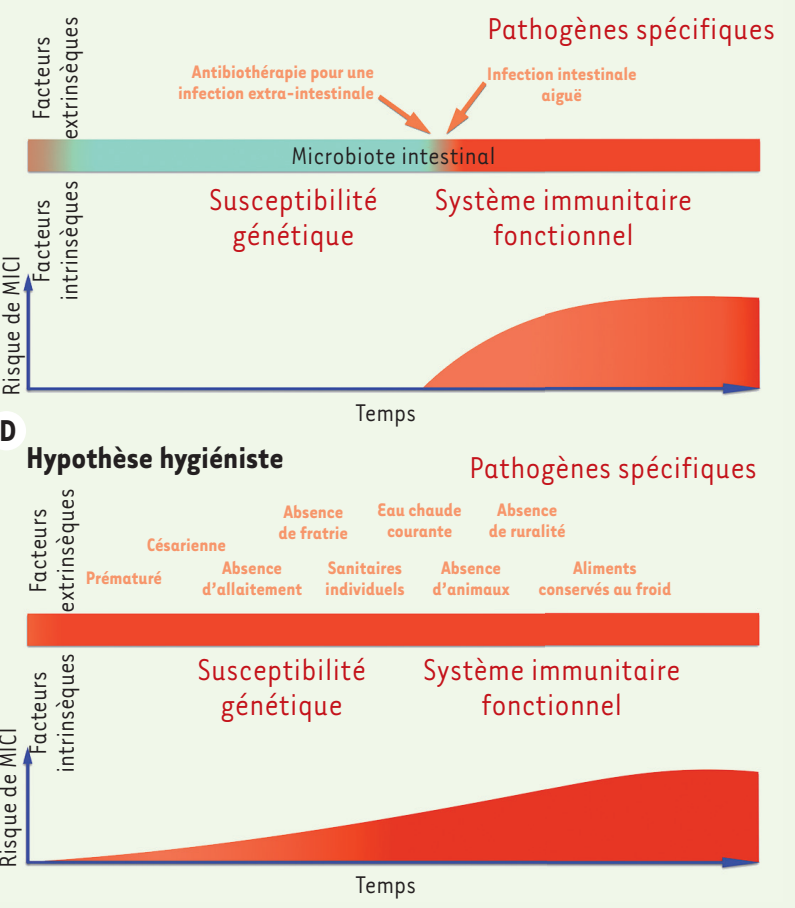

Figure 2. Modification du microbiote intestinal avant le déclenchement d'une maladie inflammatoire chronique de l'intestin (MICI). A. Chez l'individu en eubiose, une protection contre les états pathologiques existe avec un risque faible de développer une $\mathrm{MICl}$. B. Un régime alimentaire « occidental » conduit à une dysbiose et, progressivement, à un risque plus élevé de MICI. C. Une infection gastro-intestinale aiguë et des antibiothérapies favorisent une dysbiose et augmentent le risque de développement des $\mathrm{MICl}$. D. L'hypothèse de l'hygiène, qui propose un contact réduit avec une grande variété de micro-organismes, entraîne une dysbiose intestinale dès la petite enfance et est associée à un risque élevé de MICI.

À l'inverse du régime «occidentalisé », une consommation élevée en fibres alimentaires, en fruits et en légumes a un effet protecteur dans les $\mathrm{MICl}$. Le mécanisme par lequel les fruits et les légumes confèrent une protection contre la maladie serait en lien avec leur capacité de modifier l'activité des enzymes ${ }^{3}$ impliquées dans l'élimination des espèces réactives de l'oxygène (ROS) [3].

Le butyrate, produit par les bactéries, joue un rôle de régulateur sur le transport transépithélial de fluides. De faibles quantités de butyrate pourraient être impliquées dans l'initiation et/ou la perpétuation de I'inflammation. Le butyrate réduit l'inflammation des muqueuses et le statut oxydatif. Au niveau de l'intestin, il a un rôle essentiel : il entraîne une diminution du pH intraluminal, favorise le développement des bactéries commensales, est une source d'énergie pour les cellules du côlon, maintient la fonction de barrière épithéliale, et exerce des activités immunomodulatrices, anti-inflammatoires, et anticancéreuses. II stimule les histone désacétylases (HDAC), des enzymes intervenant dans la régulation de la transcription, et l'activité des

\footnotetext{
${ }_{3}^{3}$ Par exemple, les enzymes de la famille des glutathione S-transférases participent aux processus de détoxification au niveau de l'intestin. Les fruits et légumes comportent des constituants qui augmentent l'expression et l'activité de ces enzymes.
}

PPAR (peroxisome proliferator-activated receptor) qui inhibent les cytokines pro-inflammatoires et activent les cellules $T$ régulatrices, par les récepteurs couplés aux protéines $G$ $($ GPR) 43 et 109a [26] $\rightarrow$ ).

$(\rightarrow)$ Voir la Synthèse de A. Stetdman, et al., page 983 II régule également la production de ce numéro d'IL-10, réduit l'expression de cytokines liées au TNF $\alpha$ (tumor necrosis factor alpha), et inhibe I'IL-12, possiblement en interférant avec la voie de signalisation $\mathrm{NF}-\kappa \mathrm{B}$ (nuclear factor-kappa B) et l'expression de STAT-3 (signal transducer and activator of transcription 3) [9].

Certains aliments sembleraient donc bénéfiques, ou néfastes, au maintien de l'eubiose. Une alimentation déséquilibrée, riche en graisses et en sucres, pourrait conduire à une dysbiose intestinale et augmenter le risque de développer une $\mathrm{MICl}$ (Figure 2B).

La perte de microbes bénéfiques, et par conséquent des produits qu'ils synthétisent, peut ainsi permettre l'émergence de microbes pathogènes qui, eux, produisent des métabolites présentant des effets négatifs 
sur l'intestin, en condition inflammatoire. Ainsi, les patients atteints de RCH ont, dans leur microbiote intestinal, des taux élevés de bactéries réduisant le soufre et de bactéries tolérant la bile, avec une augmentation concomitante de la production de substances toxiques comme le sulfure d'hydrogène qui a une activité pro-inflammatoire [11].

\section{La rupture aiguë de l'eubiose}

Le risque de $\mathrm{MICl}$ peut être augmenté après une rupture aiguë de l'eubiose. Deux principaux mécanismes peuvent induire une perturbation soudaine de la microflore normale: une infection gastro-intestinale aiguë (IGA) ou une antibiothérapie (même pour le traitement d'une infection extra-intestinale) (Figure 2C).

\section{L'infection gastro-intestinale}

L'exposition du système immunitaire intestinal aux infections semblerait jouer un rôle dans le développement de ces maladies chroniques qui sont très fréquentes chez le jeune. Ainsi, entre 5 et $30 \%$ des patients qui ont souffert d'un épisode aigu de gastro-entérite infectieuse développent des symptômes gastro-intestinaux chroniques, malgré l'élimination de l'agent pathogène à l'origine de I'infection $[12,13]$. Le taux d'incidence de $\mathrm{MICl}$ a ainsi été estimé à 68,4 pour 100000 chez des personnes ayant présenté, quelques années auparavant, un épisode de gastro-entérite infectieuse aiguë par rapport à celui observé dans une cohorte d'individus n'ayant pas été infectés qui est de 29,7 pour 100000 [13]. Des études ont suggéré que les IgA (immunoglobulines $A$ ) induites à la suite d'une infection par Salmonella ou Campylobacter pouvaient initier le processus conduisant à la $\mathrm{MICI}$ [12]. Pour la MC, qui se produit souvent après une gastro-entérite infectieuse, on retrouve une diversité de la flore au niveau des muqueuses et un nombre augmenté de bactéries présentant souvent des propriétés adhésives [14].

\section{L'antibiothérapie : une action à distance}

L'exposition aux antibiotiques durant la petite enfance, quand l'eubiose n'est peut-être pas encore totalement établie, est supposée interférer avec le processus normal de développement de tolérance aux bactéries entériques (Figure 2C) [3]. De nombreuses études soulignent le possible rôle des antibiotiques parmi les facteurs de risque de développement des $\mathrm{MICl}$.

Une analyse prospective canadienne a en effet montré un risque relatif élevé de développer des $\mathrm{MICl}$ chez les patients ayant reçu un traitement antibiotique au cours des années précédant le diagnostic (4 à 5 ans) [15].

L'utilisation des antibiotiques serait associée à un fort risque de développer une MC. Cela se traduirait par une diminution du nombre de bactéries bénéfiques (comme les bactéries du genre Bifidobacterium, Lactobacillus, Bacteroides et Firmicutes) et par une augmentation du nombre de bactéries pathogènes, comme les AIEC (Escherichia coli adhérent et invasif) qui ont la capacité d'adhérer et d'envahir l'épithélium intestinal.
L'hypothèse de l'hygiène : une microflore déséquilibrée

L'hypothèse de l'hygiène est définie comme une exposition insuffisante, durant l'enfance, à des agents infectieux. Elle favoriserait le développement des $\mathrm{MICl}$ (Figure 2D). Cette hypothèse propose que la fréquence croissante des troubles immunologiques de l'adulte que l'on observe actuellement puisse être attribuée à un manque d'exposition dans l'enfance à une grande variété de microorganismes. L'amélioration de l'assainissement et de l'hygiène (disponibilité de l'eau potable filtrée et de l'eau chaude, hygiène domestique et conditions sanitaires alimentaires) vécue par l'enfant, peut ainsi conduire au développement, plus tard dans la vie, d'une réponse immunologique vis-à-vis de nouveaux antigènes (lors d'une infection par exemple) qui est inappropriée [3]. Vivre en milieu urbain (versus en milieu rural), et avoir un statut socioéconomique élevé seraient également des facteurs de risque de développer une $\mathrm{MICl}$. L'association entre $\mathrm{MICl}$ et hypothèse de l'hygiène pourrait provenir de l'absence d'éducation du système immunitaire à devenir tolérant aux microorganismes faute d'avoir été exposé à des organismes variés [3].

L'allaitement maternel peut réduire le risque de développer une $\mathrm{MICl}$. Il est important pour l'acquisition de la tolérance orale à la microflore et aux antigènes alimentaires. Le mécanisme par lequel l'allaitement peut avoir un effet bénéfique est sans doute multifactoriel. À noter que les préparations pour nourrissons, contrairement au lait maternel, ne contiennent pas de lactoferrine, une glycoprotéine ayant des activités antibactériennes, antivirales, et anti-inflammatoires [3].

\section{Des microorganismes pathogènes dans l'intestin des patients atteints de $\mathrm{MICl}$}

Chez les patients atteints de $\mathrm{MICl}$, la dysbiose peut permettre une colonisation et une croissance de microorganismes potentiellement pathogènes et pro-inflammatoires. Plusieurs agents ont été impliqués dans la MC : Candida albicans, Listeria monocytogenes, Mycobacterium avium sous-espèce paratuberculosis (MAP), Bacteroides fragilis produisant une entérotoxine et Escherichia coli adhérent et invasif (AIEC).

\section{Candida albicans}

Des anticorps anti-levures, mis en évidence par des tests utilisant des antigènes glycaniques isolés de la levure Saccharomyces cerevisiae (ASCA), sont détectés chez 50 à $60 \%$ des patients présentant une MC et, de façon intéressante, chez 20 à $25 \%$ des membres sains 
de leur famille. Candida albicans, une levure commensale du tube digestif de l'homme, peut induire la production de ces anticorps ASCA, l'antigène reconnu étant partagé par les deux levures. C. albicans est également retrouvé plus souvent dans les selles de patients atteints de MC (44\%) et dans celles des membres sains de leur famille (38\%) que chez les témoins $(22 \%)[16,27](\rightarrow)$. $(\rightarrow)$ Voir la Synthèse de B. Sendid, $m / s$ $n^{\circ} 5$, mai 2009, page 473
Cette levure est aussi à l'origine de dérèglements de la réponse immunitaire pro-inflammatoire qui la rendent aberrante [17].

\section{Listeria monocytogenes}

Listeria monocytogenes est un pathogène d'origine alimentaire responsable de la listériose, une infection souvent fatale évoluant en septicémie et méningite (surtout chez le nouveau-né). Des observations récentes indiquent que cette bactérie peut être responsable, chez la personne immunocompétente, d'une inflammation de la muqueuse évoluant en gastro-entérite fébrile. L. monocytogenes peut pénétrer soit directement la muqueuse par l'entérocyte, soit indirectement, via les plaques de Peyer [18].

\section{MAP (Mycobacterium avium sous-espèce paratuberculosis)}

Les bactéries du groupe MAP induisent, chez les ruminants, une entérocolite granulomateuse chronique qui présente une histologie similaire à celle observée dans les $\mathrm{MICl}$. Son évolution temporelle est également semblable à celle des $\mathrm{MICl}$. Ces bactéries ont été retrouvées avec une fréquence augmentée chez des patients atteints de MC, mais son rôle précis reste très controversé [5].

\section{Bacteroides fragilis et Clostridium spp}

Un sous-groupe de souches de Bacteroides fragilis, nommé ETBF (Bacteroides fragilis entérotoxinogène) sécrète une toxine de type métalloprotéase zinc-dépendante qui est pro-inflammatoire. Elle a été associée à des troubles diarrhéiques chez l'enfant et l'adulte. Ces souches ETBF ont été retrouvées chez près de $20 \%$ des patients présentant une $\mathrm{MICl}$ cliniquement active [5].

Une grande variété de toxines, et d'autres enzymes protéolytiques, qui augmentent la perméabilité de la muqueuse et permettent la pénétration en profondeur des bactéries, sont produites par des souches de Bacteroides et de Clostridium. Ces bactéries présentent des facteurs de virulence qui sont nécessaires pour coloniser l'intestin, traverser la barrière épithéliale, interagir avec les macrophages, et induire la sécrétion de cytokines pro-inflammatoires par les cellules infectées et les macrophages [19]. Même si certaines de ces toxines s'accompagnent de signes cliniques spécifiques qui ne sont pas observés dans le cas de $M C$, elles peuvent néanmoins aggraver une inflammation chronique lorsqu'elles sont associées avec d'autres bactéries [20].

\section{Escherichia coli adhérent et invasif (AIEC)}

Escherichia coli est une espèce bactérienne à Gram négatif prédominant dans l'intestin de l'individu en bonne santé où elle participe à son l'homéostasie. Par l'acquisition de facteurs de virulence, elle peut devenir pathogène sous différentes formes appelées pathovars $^{4}[21]$.

Les patients atteints de $\mathrm{MICl}$ sont colonisés avec une fréquence anormale par des souches d' $\varepsilon$. coli présentant des capacités d'adhérence et d'invasion qui sont absentes chez les $\varepsilon$. coli commensaux. Ces souches sont capables de s'adapter et de coloniser de nouvelles niches pour ainsi participer au processus pathologique et aggraver l'inflammation. Des souches d' $\varepsilon$. coli isolées de la muqueuse intestinale de patients atteints de $M C$ peuvent en effet adhérer massivement à la muqueuse et pénétrer l'entérocyte. Selon les caractéristiques pathogéniques de ces souches, un nouveau pathovar, nommé AIEC pour $\varepsilon$. coli adhérent et invasif, a été désigné. Ces souches sont retrouvées au niveau de l'iléon de $36,4 \%$ des patients avec MC contre seulement chez $6 \%$ des témoins [22]. Elles adhèrent à I'entérocyte via des interactions qui impliqueraient les pili de type $1^{5}$ et CEACAM6 (carcinoembryonic antigenrelated cell adhesion molecule 6 ) qui est anormalement exprimé au niveau de la muqueuse iléale en cas de MC. La prévalence des AIEC dans la MC pourrait provenir de l'incapacité de la muqueuse intestinale à contrôler la colonisation par la bactérie, liée sans doute à un défaut de fonction des cellules de Paneth et de sécrétion des peptides antimicrobiens. Cette perte de contrôle pourrait également être liée à des défauts touchant les processus d'autophagie qui limitent la multiplication des microorganismes intracellulaires, des mutations des gènes impliqués dans ce mécanisme tels que NOD2 (nucleotide-binding oligomerization domain 2), ATG16L1 (autophagy related 16 like 1) ou IRGM (immunity-related GTPase $M$ ), étant fréquemment retrouvées chez les patients [22].

\section{Conclusion}

Même si la cause des $\mathrm{MICl}$ reste inconnue, beaucoup d'observations suggèrent qu'une dysbiose précède le déclenchement de la maladie. Une étude récente impliquant les membres sains de familles présentant plusieurs cas de MC, indique que chez une proportion de ces individus, il existe une augmentation de la perméabilité intestinale, une élévation de la calprotectine ${ }^{6}$ fécale, une sérologie antimicrobienne positive, des altérations

\footnotetext{
${ }^{4}$ Groupes clonaux au sein d'une espèce bactérienne produisant des facteurs de virulence faisant défaut chez les souches commensales.

${ }^{5}$ Les pili de type 1 sont des fimbriae liant les résidus mannose. Ils permettent aux bactéries de se déplacer et d'adhérer à un substrat.

${ }^{6}$ La calprotectine que l'on retrouve dans les selles, provient de sa sécrétion principalement par les polynucléaires neutrophiles recrutés et activés dans la lumière du tube digestif. L'élévation de son taux dans les selles est le témoin d'une inflammation intestinale.
} 
des marqueurs de l'immunité innée ou acquise, une composition bactérienne fécale modifiée, ou une combinaison de plusieurs de ces facteurs [23]. La susceptibilité génétique à la maladie est l'un des facteurs de risque mais des facteurs environnementaux ou alimentaires qui peuvent aggraver la dysbiose augmentent ce risque de déclencher la maladie. La dysbiose ainsi induite peut également favoriser la colonisation intestinale par des bactéries dont le potentiel pathogène est plus élevé que celui des bactéries présentes en eubiose, et ainsi augmenter l'inflammation. $\diamond$

\section{SUMMARY}

\section{Enteric microflora in inflammatory bowel disease patients}

During the last years, the importance of a well equilibrated intestinal microbiota (eubiosis) has become more and more obvious in human health. Dysbiosis is now a well-recognized feature associated with IBD (inflammatory bowel disease). Rupture of the normal microbiota can occur through different mechanisms: (1) by a typical Western diet rich in fat and low in fiber, (2) by an acute disruption of the microbiota (by an acute gastroenteritis or by intake of antibiotics) or (3) by a combination of event in early childhood avoiding the establishment of eubiosis (the hygiene hypothesis). Risk factors for IBD are stated for each disruption mechanism. Dysbiosis can also induce colonization by several pathobionts able to aggravate inflammation. Among the potential candidates in IBD, most attention has been paid on AIEC during the last years. $\diamond$

\section{LIENS D'INTÉRÊT}

Les auteurs déclarent n'avoir aucun lien d'intérêt concernant les données publiées dans cet article.

\section{RéFÉRENCES}

1. Nitzan 0 , Elias $M$, Peretz $A$, et al. Role of antibiotics for treatment of inflammatory bowel disease World J Gastroenterol 2016 ; 22 : 1078-87.

2. Dutta AK, Chacko A. Influence of environmental factors on the onset and course of inflammatory bowel disease. World J Gastroenterol 2016 ; 22 : 1088-100.

3. Molodecky NA, Kaplan GG. Environmental risk factors for inflammatory bowel disease. Gastroenterol Hepatol $2010 ; 6: 339-46$.

4. Ananthakrishnan AN. Epidemiology and risk factors for IBD. Nat Rev Gastroenterol Hepatol 2015 ; $12: 205-17$.

5. Sartor RB, Mazmanian SK. Intestinal microbes in inflammatory bowel diseases. Am J Gastroenterol $2012 ; 1$ (suppl) : 15-21.

6. Morgan XC, Tickle TL, Sokol H, et al. Dysfunction of the intestinal microbiome in inflammatory bowel disease and treatment. Genome Biol 2012 ; 13 : 1-18.

7. Comito D, Cascio A, Romano C. Microbiota biodiversity in inflammatory bowel disease. Ital J Pediatr $2014 ; 40: 1-6$
8. Leone V, Chang $\varepsilon B$, Devkota S. Diet, microbes, and host genetics: the perfect storm in inflammatory bowel diseases. J Gastroenterol $2013 ; 48: 315-21$.

9. Serban DE. Microbiota in inflammatory bowel disease pathogenesis and therapy: is it all about diet? Nutr Clin Pract $2015 ; 30: 760-79$.

10. Neuman MG, Nanau RM. Inflammatory bowel disease: role of diet, microbiota, life style. Transl Res $2012 ; 160: 29-44$.

11. Dalal SR, Chang EB. The microbial basis of inflammatory bowel diseases. J Clin Invest $2014 ; 124:$ 4190-96.

12. Jess T, Simonsen J, Nielsen NM, et al. Enteric Salmonella or Campylobacter infections and the risk of inflammatory bowel disease. Gut $2011 ; 60$ : 318-24.

13. Rodríguez LAG, Ruigómez A, Panés J. Acute gastroenteritis is followed by an increased risk of inflammatory bowel disease. Gastroenterology 2006 ; 130 1588-94.

14. Baumgart DC, Sandborn WJ. Crohn's disease. Lancet 2012 ; 380 : 1590-605.

15. Shaw SY, Blanchard JF, Bernstein CN. Association between the use of antibiotics and new diagnoses of crohn's disease and ulcerative colitis. Am J Gastroenterol $2011 ; 106: 2133-42$.

16. Standaert-Vitse A, Sendid B, Joossens M, et al. Candida albicans colonization and ASCA in familial Crohn's disease. Am J Gastroenterol 2009 ; $104: 1745-53$.

17. Standaert-Vitse A, Jouault $T$, Vandewalle $P$, et al. Candida albicans is an immunogen for anti-Saccharomyces cerevisiae antibody markers of crohn's disease. Gastroenterology 2006 ; 130 : 1764-75.

18. Schuppler M, Loessner MJ. The opportunistic pathogen Listeria monocytogenes: pathogenicity and interaction with the mucosal immune system. Int J Inflam 2010; $2010: 1-12$.

19. Tamboli CP, Neut C, Desreumaux P, et al. Dysbiosis in inflammatory bowel disease. Gut $2004 ; 53: 1-4$.

19. Drouet $M$, Vignal $C$, Singer $\varepsilon$, et al. AlEC colonization and pathogenicity: influence of previous antibiotic treatment and preexisting inflammation. Inflamm Bowel Dis 2012; 18 : 1923-31.

20. Sears CL. Enterotoxigenic Bacteroides fragilis: a rogue among symbiotes, Clin Microbiol Rev $2009 ; 22$ : 349-69.

21. Small CL, Reid-Yu SA, McPhee JB, et al. Persistent infection with Crohn's disease-associated adherent-invasive Escherichia coli leads to chronic inflammation and intestinal fibrosis. Nat Common 2013 ; 4 : 1-19.

22. Chassaing B, Darfeuille-Michaud A. The commensal microbiota and enteropathogens in the pathogenesis of inflammatory bowel diseases. Gastroenterology $2011 ; 140: 1720-8$.

23. Torres J, Burisch J, Riddle M, et al. Preclinical disease and preventive strategies in IBD: perspectives, challenges and opportunities. Gut 2016; 65 : 1061-9.

24. Burcelin R, Nicolas S, Blasco-Baque V. Microbiotes et maladies métaboliques: de nouveaux concepts pour de nouvelles stratégies thérapeutiques. Med Sci (Paris) $2016 ; 32:$ 952-60.

25. Gaboriau-Routhiau V, Cerf-Bensussan N. Microbiote intestinal et développement du système immunitaire. Med Sci (Paris) 2016 ; 32 : 961-7.

26. Stetdman A, Nigro G, Sansonetti P. Le dialogue microbiote-cellules souches: un élément clé pour la régénération intestinale. Med Sci (Paris) 2016; 32 : 983-90.

27. Sendid B, Jouault T, Vitse A. Glycannes pariétaux de levures et anticorps spécifiques. Med Sci (Paris) 2009 ; $25:$ 473-82.

\section{TIRÉS À PART}

C. Neut

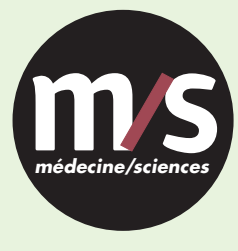

Tarifs d'abonnement $m / s-2016$

Abonnez-vous

à médecine/sciences
$>$ Grâce à $m / s$, vivez en direct les progrès des sciences biologiques et médicales

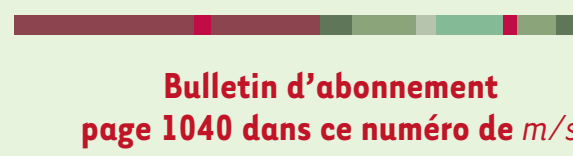

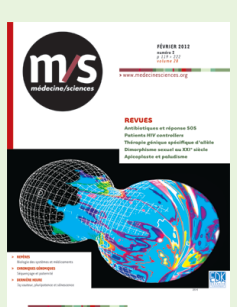

